\title{
The influence of culture media on chromosome aberration levels, sister chromatid exchange frequencies, and the rate of cell proliferation: comparison of Iscove's low folate medium with Eagle's MEM
}

The use of a low folate culture medium has been recognised as essential for the expression of fragile sites on human chromosomes and has been widely adopted in clinical cytogenetics. For technical reasons it was proposed that the use of serum free Iscove's low folate medium be extended to all the lymphocyte cultures in this laboratory, including population monitoring of exposure to possible clastogens. However, the type of culture medium used can affect the frequencies of chromosome breakage, ${ }^{1}$ sister chromatid exchange (SCE) ${ }^{2}{ }^{3}$ and also the cell proliferation rate. ${ }^{23}$

This study was initiated to compare the effects of Iscove's low folate medium with Eagle's MEM plus fetal bovine serum on the endpoints generally studied in this laboratory, that is, chromosome aberrations in first division metaphases from 48 hour cultures and sister chromatid exchange frequency in second division metaphases from 72 hour cultures. The rate of cell cycle progression was also examined.

\section{Methods}

Peripheral blood samples were obtained from 10 healthy, male, new entrants to the company. Two culture media were used: Eagle's MEM (Wellcome), containing $1.0 \mathrm{mg} / \mathrm{l}$ folic acid and supplemented with $15 \%$ fetal bovine serum, and Iscove's low folate medium (Imperial Labs), containing $0.01 \mathrm{mg} / \mathrm{l}$ folic acid. Penicillin and streptomycin were present in the Eagle's MEM and kanamycin was added to the Iscove's medium. The cultures were stimulated with phytohaemagglutinin and bromodeoxyuridine was present throughout at a concentration of $10 \mu \mathrm{mol} / \mathrm{l}$. Colcemid was added for the final four hours of culture. Duplicate cultures were harvested at 48 hours and 72 hours.

Received for publication 12 May 1987.

Revised version accepted for publication 6 July 1987.
Slides were stained using a fluorescence plus Giemsa technique. Two hundred metaphases in their first mitosis in vitro from 48 hour cultures were analysed for asymmetrical chromosome and chromatid aberrations. SCE frequency was determined from 30 cells in second mitosis from 72 hour cultures (15 from each duplicate culture). The proportion of cells in first, second, and third+ mitoses at 72 hours was evaluated by taking the mean of 100 cells from each duplicated culture.

\section{Asymmetrical aberrations}

Analysis of first division metaphases from 48 hour cultures for asymmetrical aberrations revealed no difference in the total aberration frequencies nor in the frequencies of the different aberration types for the two media (table).

Although many previous reports have shown significant increases in aberrations, particularly breaks and gaps, using low folate medium, these have involved longer culture times of 72 hours and 96 hours. An increase in breakage with increased culture time has been shown ${ }^{4}$ and a similar time dependence has been reported for fragile X expression. ${ }^{5}$ By confining the analysis to cells in their first mitosis in vitro it is likely that the intracellular levels of fo'ic acid are still sufficiently high for DNA replication to remain unaffected, so preventing any manifestations of fragility.

\section{SCEs and cell proliferation rate}

In nine of the 10 subjects higher levels of SCEs were found using Iscove's low folate medium compared with Eagle's MEM $(t=3.48, \mathrm{df}=9, \mathrm{p}<0.01)$ (table). In addition, these nine had slower cell cycle progression times, as seen by the lower percentage of cells in third+ division, with Iscove's medium. However, one subject had a faster progression time with Iscove's and a lower level of SCEs. Thus, a 
consistent relationship between cell proliferation rate and SCE frequency was found for all 10 subjects.

An effect of different culture media on SCE frequency has been widely reported. However, two such studies suggested that it was changes in the cell proliferation rate and not a direct effect on SCE production that gave rise to changes in the observed frequencies of SCEs. ${ }^{23}$

Conflicting results on the effect of different

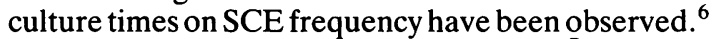
A more recent detailed study of controls, ${ }^{7}$ found an increase in SCEs from 54 to 68 hours, a decrease to 72 hours, and a subsequent increase to 78 hours, supporting an earlier suggestion ${ }^{8}$ that the differences in SCE levels expressed within control subjects are due to different sub-populations of lymphocytes with different spontaneous SCE levels and proliferation rates.

Thus, in the present study, for nine of the 10 subjects a faster dividing population of cells was sampled at 72 hours with Iscove's low folate medium, whereas with Eagle's MEM many of these cells will have already entered third division and it is the more slowly dividing ones that are now in second division. With one subject the reverse was found, with the faster dividing cells sampled with Eagle's MEM and the slower ones with Iscove's.

No attempt has been made in this short note to correlate the change in SCE level with the change in cell proliferation rate for the group as a whole, since interaction with other variables, for example, age and smoking, may well cause differences between persons. Further work with the two media using a range of harvesting times might elucidate the situation. However, the study illustrates the importance of appreciating that changes in SCE frequency may simply reflect changes in cell proliferation and are not necessarily indicative of genotoxicity.

\section{E JANET TAWN AND R EARL \\ The Geoffrey Schofield Cytogenetics Laboratory, British Nuclear Fuels plc, Sellafield, Cumbria CA20 IPG.}

\footnotetext{
References

' Reidy JA, Zhou X, Chen ATL. Folic acid and chromosome breakage. I. Implications for genetoxicity studies. Mutat Res 1983;122:217-21.

2 Das BC, Sharma T. Reduced frequencey of base-line sister chromatid exchanges in lymphocytes grown in antibiotics and serum-excluded culture medium. Hum Genet 1983;64:249-53.

${ }^{3}$ Lamberti L, Ponzetto PB, Ardito G. Cell kinetics and sisterchromatid-exchange frequency in human lymphocytes. Mutat Res 1983;120:193-9.

4 Reidy JA, Chen ATL. Folic acid and chromosome breakage. II. A methionine effect similar to that in fragile X expression. Hum Genet 1984;68:189-90.
} 
5 Sutherland GR. The fragile X chromosome. Int Rev Cytol 1983;81:107-43.

${ }^{6}$ Obe G, Beek B. Human peripheral lymphocytes in mutation research. In: Obe G, ed. Mutations in man. Berlin: SpringerVerlag, 1984:177-97.

7 Parkes DJG, Scott D, Steward B. Changes in spontaneous SCE frequencies as a function of sampling time in lymphocytes from normal donors and cancer patients. Mutat Res 1985;147:113-22.

${ }^{8}$ Snope AJ, Rary JM. Cell-cycle duration and sister-chromatid exchange frequency in cultured human lymphocytes. Mutat Res 1979;63:345-9.

Correspondence and requests for reprints to $\mathrm{Dr} E \mathrm{~J}$ Tawn, The Geoffrey Schofield Cytogenetics Laboratory, British Nuclear Fuels plc, Sellafield, Cumbria CA20 1PG. 